\title{
Dynamics of Second-Harmonic Generation in a Plasmonic Silver Nanorod
}

\author{
Gabriel D. Bernasconi, ${ }^{*(1)}$ Jérémy Butet, $^{\left({ }^{\circ}\right.}$ and Olivier J. F. Martin ${ }^{(1)}$ \\ Nanophotonics and Metrology Laboratory, Swiss Federal Institute of Technology Lausanne (EPFL), 1015 Lausanne, Switzerland
}

Supporting Information

ABSTRACT: Second-harmonic generation in plasmonic nanostructures is known to enable the observation of modes with vanishing dipolar moments, i.e., having small radiation losses and thus long lifetimes. With the aid of a full wave numerical method, we study the far-field temporal dynamics of the linear and nonlinear responses of a silver nanorod driven by femtosecond pulses. The results show that the plasmons lifetime is observable in the decaying field oscillations surviving after the exciting pulse, for both processes, and fits with the

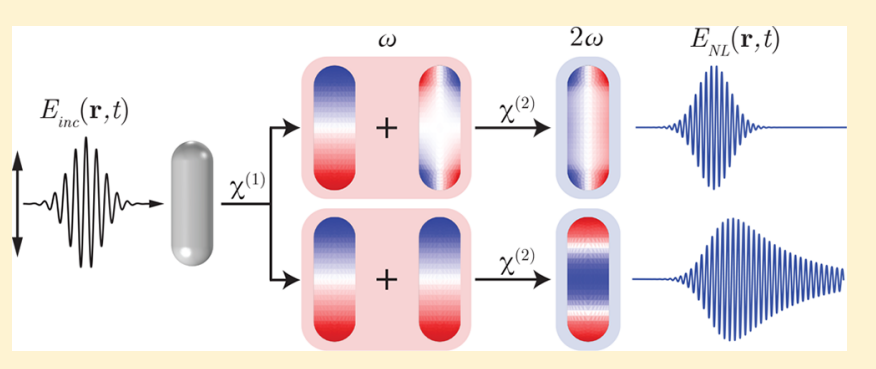
damped harmonic oscillator model. In addition, using a detailed mode analysis, we find that the multipolar characteristic of the nonlinear radiation is strongly influenced by both the pulse central frequency and width. Implications for the accurate measurement of plasmon lifetime with the help of nonlinear optics are discussed, especially the need to carefully disentangle the linear and nonlinear plasmon dynamics.

KEYWORDS: SHG, SFG, eigenmodes, multipoles, nanoparticles, surface integral equation method, ultrafast plasmonics

$\mathrm{P}$ lasmonic nanoparticles are well known for their ability to confine light below the diffraction limit. ${ }^{1,2}$ As a consequence of this light concentration, the electromagnetic field intensity can be locally enhanced by several orders of magnitude. ${ }^{1-3}$ These localized surface plasmon resonances are versatile, and their properties depend upon several parameters, such as the nanostructure shape, material, surrounding medium, and the coupling between nanoparticles. ${ }^{4,5}$ Among the different parameters that describe a plasmon resonance, the lifetime is of prime importance, since it controls the field enhancement. ${ }^{6}$ Furthermore, the coherent control of the field localization in both time and space is intrinsically linked to the plasmon lifetime. ${ }^{7-10}$ However, the plasmon damping time is very short (10 fs and below) and is thus difficult to measure experimentally. ${ }^{11,12}$

Recently, time-resolved photoemission electron microscopy has emerged as a useful approach for studying plasmon dynamics, allowing the detection of electron oscillations at the nanoscale. ${ }^{13-15}$ In this context, optical techniques used for the characterization of ultrashort laser pulses have been extended for the investigation of the ultrafast response of plasmonic systems, ${ }^{16-18}$ including nonlinear effects. ${ }^{19}$ These optical measurements are based on interferometry and rely on the evaluation of the autocorrelation function. This is generally performed by recording the nonlinear light conversion, either second-harmonic generation $(\mathrm{SHG})^{16-18}$ or third-harmonic generation, ${ }^{20,21}$ as a function of the time delay between the light traveling through the two branches of an interferometer. A broadening of the autocorrelation function, in comparison with a nonresonant reference (as a BBO crystal for example), is indeed caused by the field enhancement induced by the free electron collective oscillations.

During the past few years, particular attention has been devoted to the SHG from plasmonic nanostructures, ${ }^{22,23}$ and SHG from plasmonic nanostructures with various shapes and properties has been reported. ${ }^{24-30}$ A key recent advance in this field has been the design of double-resonant nanostructures to boost the nonlinear optical conversion at the nanoscale. ${ }^{31-35}$ These results emphasize the importance of the plasmonic modes at both the fundamental and second-harmonic $(\mathrm{SH})$ frequencies in the overall nonlinear optical conversion process. To investigate the relation between the modes at the excitation and emission wavelengths in detail, it was recently proposed to combine eigenmode analysis with the evaluation of the SHG. ${ }^{36-38}$ However, the distinct plasmon dynamics at the fundamental and $\mathrm{SH}$ frequencies have not been addressed so far, despite important implications for the coherent control of nonlinear radiation, as well as for the accurate measurement of the plasmon lifetime using nonlinear optical processes.

In this article, we study numerically the second-order nonlinear response of a silver nanorod driven by femtosecond pulses under plane-wave illumination. We use a frequency domain surface integral equation (SIE) method to compute the linear and nonlinear fields as well as the eigenmodes of the silver nanorod. We show that single-eigenmode dynamics behaves like a damped harmonic oscillator, ${ }^{12}$ in both the linear and nonlinear regimes. A multipolar analysis of the timedependent far-field intensity for different pulse central

Received: April 12, 2018

Published: May 18, 2018 


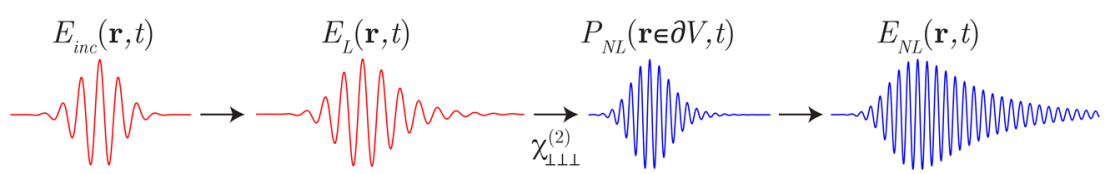

Figure 1. Schematized computation of the SFG temporal signals. The incoming pulse $E_{\text {inc }}(\mathbf{r}, t)$ excites the structure and generates the linear response $E_{\mathrm{L}}(\mathbf{r}, t)$. The linear response is used to obtain the nonlinear surface polarization $P_{\mathrm{NL}}(\mathbf{r} \in \partial V, t)$ at the nanostructure boundary $\partial V$. Finally, these nonlinear sources are used to compute the scattered nonlinear field $E_{\mathrm{NL}}(\mathbf{r}, t)$. This sketch illustrates the SFG process in the time domain, although each computation step is performed in the frequency domain (see text).

frequencies and widths is also conducted, revealing the importance of the modal structure to interpret the radiated signals at the $\mathrm{SH}$ wavelength.

\section{THEORY}

Numerical simulations are made using a frequency domain full wave method, namely, the surface integral equation. ${ }^{39}$ Only the surface of the nanorod is discretized with triangular elements, and the scattering problem is solved by enforcing the boundary conditions on the fields at the nanostructure surface. Harmonic oscillations of the form $\mathrm{e}^{-i \omega t}$ are assumed throughout this article, with $i=\sqrt{-1}$. The angular frequency $\omega$ has $\mathrm{rad} \cdot \mathrm{s}^{-1}$ units, but we use electronvolts $(\mathrm{eV})$ to express it, i.e., $\omega=h \nu / q_{0}$ with $\nu$ the frequency in $\mathrm{Hz}, h$ Planck's constant, and $q_{0}$ the elementary charge.

A temporal signal with a finite duration is necessarily composed of more than one frequency, and the second-order nonlinearity will lead to sum-frequency generation (SFG) in addition to SHG. ${ }^{40}$ Neglecting optical rectification $\left(\omega_{m}-\omega_{m}\right)$ and difference-frequency generation $\left(\omega_{m}-\omega_{n}\right)$, the secondorder nonlinear polarization $P^{(2)}(t)$ due to the complex harmonic fields $E_{1}(t)$ and $E_{2}(t)$ of the form $E_{m}(t)=$ $E_{m}\left(\omega_{m}\right) \mathrm{e}^{-i \omega_{m} t}$ is ${ }^{40}$

$$
P^{(2)}(t)=P_{2 \omega_{1}} \mathrm{e}^{-i 2 \omega_{1} t}+P_{2 \omega_{2}} \mathrm{e}^{-i 2 \omega_{2} t}+P_{\omega_{1}+\omega_{2}} \mathrm{e}^{-i\left(\omega_{1}+\omega_{2}\right) t}
$$

with $P_{2 \omega_{1}}=\epsilon_{0} \chi^{(2)} E_{1}^{2}, P_{2 \omega_{2}}=\epsilon_{0} \chi^{(2)} E_{2}^{2}$, and $P_{\omega_{1}+\omega_{2}}=2 \epsilon_{0} \chi^{(2)} E_{1} E_{2}$. The first two terms in eq 1 correspond to SHG and the last one to SFG.

The second-order nonlinearity is assumed to come uniquely from the surface of the nanoparticle where the centrosymmetry of the silver crystal is effectively broken. Furthermore, we consider only the $\chi_{\perp \perp \perp}^{(2)}$ component of the second-order susceptibility tensor, ${ }^{41-43}$ the subscript $\perp$ referring to the component normal to the surface. In the following, the method described in refs 44 and 45 to compute SHG is modified to also compute the nonlinear polarization due to SFG. We consider that the frequency range of interest is far from any electronic resonances in silver and that the second-order process is parametric, thus leading to a $\chi_{\perp \perp \perp}^{(2)}$ that is real and frequency independent. ${ }^{40}$ Furthermore, because no comparison is made between the relative amplitude of the linear and nonlinear fields, the value of the nonlinear susceptibility relatively to the linear susceptibility is not relevant, and we set $\chi_{\perp \perp \perp}^{(2)}=1$ without loss of generality. Additionally, we make the undepleted pump approximation and neglect the energy transfer of second-order waves back to fundamental waves.

The SFG computation can be schematized in four steps in the time domain, as shown in Figure 1. First the incoming pulse $E_{\text {inc }}$ excites the system and the resulting linear response $E_{\mathrm{L}}$ is computed. This linear response is then used to create the nonlinear sources $P_{\mathrm{NL}}$ at the surface, through the nonlinear susceptibility $\chi_{\perp \perp \perp}^{(2)}$. Those nonlinear sources in turn excite the system at the nonlinear frequencies to give the nonlinear fields $E_{\mathrm{NL}}$. Throughout this article $P_{\mathrm{NL}}$ is referred to as the nonlinear excitation; the color red is used for spectra at the excitation wavelength, while blue is used for the nonlinear response of the system.

In the small-particle limit, the two main contributions to SHG are expected to be due to electric quadrupolar and dipolar modes, noted $E_{2}$ and $E_{1}$, the latter being allowed by retardation effects. ${ }^{46}$ Using the standard excitation-radiation schematic notation, ${ }^{47}$ these processes are written $E_{1}+E_{1} \rightarrow E_{2}$ and $E_{1}+$ $E_{2} \rightarrow E_{1}$, where the symbols on the left and right refer to the nature of the modes respectively at the fundamental and nonlinear stages. The exact nature of the nanorod modes, transverse or longitudinal, also has to be detailed here. The nonlinear quadrupolar emission comes from the interaction of two dipoles, whereas the dipolar nonlinear emission comes from the interaction of a dipole and a quadrupole, both at the fundamental stage.

Ohmic and radiative losses are both present in plasmonic systems; thus the plasmon oscillations are damped over time and each eigenmode is characterized by a complex eigenfrequency $\omega_{\mathrm{c}}=\omega_{\mathrm{r}}+i \omega_{i}$. If no excitation is present, any quantity $A$ associated with one eigenmode (field, charge, current, ...) evolves according to the damped harmonic oscillator equation. Indeed, since the computations are done in the frequency domain, the temporal evolution is of the form $A(t) \propto \operatorname{Re}\left\{\mathrm{e}^{-i \omega t}\right\}$. Replacing $\omega$ by $\omega_{\mathrm{c}}$ leads to

$$
A(t) \propto \cos \left(\omega_{\mathrm{r}} t\right) \mathrm{e}^{\omega_{i} t}
$$

with $\omega_{i}<0$, as required for having a decaying amplitude. An example of the response of a damped harmonic oscillator to a Gaussian pulse is shown in the Supporting Information, Figure S1. Notably, there is a delay between the maximum of the driving pulse and the maximum of the response. Indeed, because of causality, the impulse response of a damped harmonic system vanishes for $t<0$ and is thus asymmetric with respect to $t=0$. The maximum of the response that is given by the convolution of the impulse response and the excitation can then only occur at a time larger that the incoming pulse maxima. Additionally, as the exciting pulse becomes longer, this delay increases, due to the fact that the system has more time to build up its response. As shown in Figure S2, this delay also increases as the central frequency of the pulse becomes close to the resonant frequency $\omega_{\mathrm{r}}$ of the oscillator and increases with the pulse temporal width $\Delta t$. This effect will be apparent in the subsequent analysis of the dynamical response of the silver nanorod.

The eigenmodes are obtained by using complex frequency excitation to find poles of the nanostructure response ${ }^{48}$ and then verified to be eigenvectors of the SIE matrix. ${ }^{37} \mathrm{An}$ analytical continuation of the permittivity function in the complex plane is needed for the eigenmode computation; thus a Drude model is used to find the eigenmode. The model is 
(a)

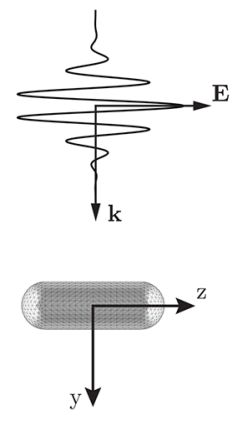

(b)

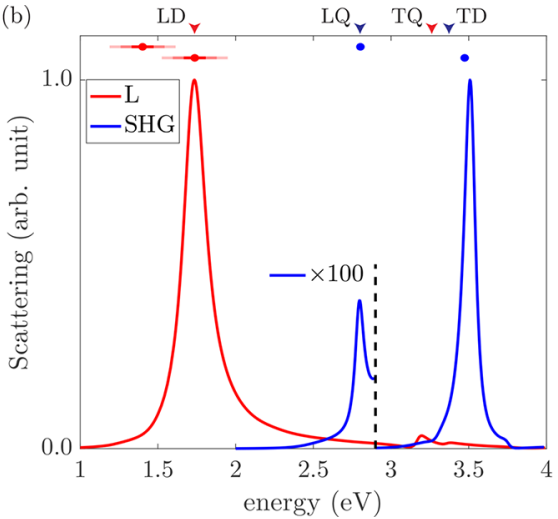

(c) Eigenmodes surface charge density $\sigma$

$-|\sigma|_{m}$

LD

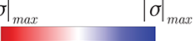

LQ
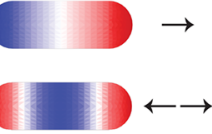

TQ

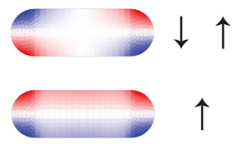

Figure 2. (a) Sketch of the excitation configuration. (b) Plane-wave scattering spectrum, linear monochromatic response (red curve), and corresponding SHG (blue curve). The SHG on the left of the vertical dashed line is multiplied by a factor 100 to highlight the resonance of the LQ mode. The red dots and horizontal lines respectively indicate the central energies $\omega_{0}$ and width $2 \Delta \omega_{0}$ at the fundamental stage for the pulses used here. The blue dots give the central frequency of the corresponding nonlinear excitation $P_{\mathrm{NL}}$ without taking into account the influence of the linear response, i.e., $\omega_{0}^{\mathrm{NL}}=2 \omega_{0}$. The position of the eigenmodes presented in (c) are shown on the top abscissa. (c) Surface charge distributions $\sigma$ of the four eigenmodes discussed here. LD stands for longitudinal dipole, LQ for longitudinal quadrupole, TD for transverse dipole, and TQ for transverse quadrupole; see Table 1. Black arrows represent the local dipolar moment orientations.

fitted to the experimental values of ref 49 in the range $1.5<\omega<$ $3 \mathrm{eV}$. The parameters of the model are $\omega_{\mathrm{p}}=9.3 \mathrm{eV}, \gamma=0.03$ $\mathrm{eV}$, and $\epsilon_{\infty}=4.3$, with $\omega_{\mathrm{p}}$ the plasma frequency, $\gamma$ the damping constant, and $\epsilon_{\infty}$ the permittivity for $\omega \rightarrow \infty$. The background is assumed to be water, with refractive index $n_{\mathrm{bg}}=1.33$, i.e., $\epsilon_{\mathrm{bg}}$ $=1.77$. This choice is made to render our result comparable to possible experimental findings, where nanoparticles are usually suspended in water or deposited on a substrate. The effective background permittivity of the latter medium depends on its composition but is indeed closer to that of water than vacuum.

For the femtosecond pulse, we consider a Gaussian pulse envelope $S(\omega)$ in the frequency domain, with width $\Delta \omega$ and centered at $\omega_{0}$,

$$
S(\omega)=\mathrm{e}^{-\left(\omega-\omega_{0}\right)^{2} / 2 \Delta \omega^{2}}
$$

The corresponding temporal signal is also a Gaussian with width $\Delta t=2 \pi / \Delta \omega$, and the full width at half-maximum is given by $\mathrm{fwhm}=2 \sqrt{2 \ln (2)} \Delta t \approx 2.35 \Delta t$. The total energy of the pulse is given by $\int|S(\omega)|^{2} \propto \Delta \omega$, so that it scales linearly with the width of the pulse, in both frequency and time domains. The construction of the temporal signal from the frequency domain computation is made using a Fourier transform as detailed in the Methods section.

\section{RESULTS}

In this section, we present the dynamics of the linear and second-order nonlinear responses of a single silver nanorod of total length $120 \mathrm{~nm}$ and diameter $40 \mathrm{~nm}$ with hemispherical ends, Figure 2(a).

We consider that the pulse excitation is built from planewaves with electric field polarization and propagation vectors respectively parallel and normal to the nanorod axis $\mathrm{Oz}$ and use the experimental values of ref 49 for the silver permittivity.

Harmonic Response and Eigenmodes. The scattering spectrum (electric field intensity integrated over the sphere) of the silver nanorod for monochromatic plane-waves incident normally to the nanorod axis is shown in Figure 2(b), as well as the corresponding $\mathrm{SH}$ spectrum. In the linear spectrum, a strong peak at $\sim 1.7 \mathrm{eV}$ is observed due to the longitudinal dipolar mode (LD). Two other features between 3.0 and $3.5 \mathrm{eV}$ also appear, due to higher order modes with a nonvanishing dipolar moment along $\mathrm{Oz}$. The $\mathrm{SH}$ spectrum reveals a first small peak at $2.80 \mathrm{eV}$ corresponding to the longitudinal quadrupole resonance (LQ) and one strong resonance at 3.5 $\mathrm{eV}$. This SHG maximum is mainly due to the dipolar resonance linearly excited at $1.74 \mathrm{eV}$ but is also expected to benefit from the transverse dipolar (TD) mode and higher order modes at the $\mathrm{SH}$ frequency. Indeed, since the real part of the permittivity becomes positive at $\omega \approx 3.8 \mathrm{eV}$, all the plasmonic modes will have resonant frequencies below $3.8 \mathrm{eV}$. From Figure 2(b), it is thus expected that some high-order modes participate in the SHG scattering due to the fact that the nonlinear sources $P_{\mathrm{NL}}$ are spectrally near $3.5 \mathrm{eV}$. A multipolar decomposition of the far field ${ }^{50}$ is also conducted for the SHG signal, Figure S3, and reveals only dipolar and quadrupolar emissions. The small peak at $2.8 \mathrm{eV}$ is composed of $85 \%$ quadrupolar emission, whereas the emission peak at $3.5 \mathrm{eV}$ is $98 \%$ dipolar. As stated in Section 2 , the SHG emission arises mainly from two excitation channels, coming from the modes presented in Figure 2(c). The first one is the interaction of the LD mode with itself to excite the LQ mode at the SH frequency. Using the previously introduced notation and specifying the precise nature of the modes, this excitation channel is $E_{1}^{\mathrm{LD}}+E_{1}^{\mathrm{LD}} \rightarrow E_{2}^{\mathrm{LQ}}$. Higher order modes with even charge distribution parity along the $\mathrm{Oz}$ axis, like the LQ mode, can be excited through identical processes involving higher order modes at the linear stage. The other excitation channel allows a dipolar emission at the $\mathrm{SH}$ frequency through the interaction of the LD and the transverse quadrupolar (TQ) mode, the latter being excited by retardation effects, i.e., phase difference of the driving field across the nanorod diameter in the $O y$ direction. This excitation channel is $E_{1}^{\mathrm{LD}}+E_{2}^{\mathrm{TQ}} \rightarrow E_{1}^{\mathrm{TD}}$. As in the case of the quadrupolar nonlinear emission, other higher order modes having the transverse characteristic, i.e., odd charge distributions along $O y$, can contribute to the dipolar nonlinear radiation through similar processes, since their symmetry does not forbid it. Figure 2(c) shows the four eigenmodes discussed above, where the arrows indicate the dipolar moments' orientations. Their complex eigenfrequencies, as well as their plasmon lifetimes and associated quality factors, are given in Table 1 . The fit of the Drude model used to obtain the eigenmodes is optimized up to 
Table 1. Eigenmode Eigenfrequencies, Lifetimes Given by $\tau$ $=h /\left(2 \pi q_{0}\left|\omega_{i}\right|\right)$, and Quality Factors $Q=\omega_{\mathrm{r}} /\left|\omega_{i}\right|^{a}$

$\begin{array}{lcccc} & \omega_{\mathrm{r}}(\mathrm{eV}) & \omega_{i}(\mathrm{eV}) & \tau(\mathrm{fs}) & Q \\ \mathrm{LD} \rightarrow & 1.74 & -0.0936 & 7.03 & 18.5 \\ \mathrm{LQ} \leftarrow \rightarrow & 2.80 & -0.0244 & 27.0 & 115 \\ \mathrm{TQ} \downarrow \uparrow & 3.27 & -0.0418 & 15.7 & 78.3 \\ \mathrm{TD} \uparrow & 3.38 & -0.168 & 3.93 & 20.1\end{array}$

${ }^{a} \mathrm{LD}$ stands for longitudinal dipole, LQ for longitudinal quadrupole, TQ for transverse quadrupole, and TD for transverse dipole. Arrows indicate the local dipolar moments as in Figure 2(c).

$3 \mathrm{eV}$; the parameters for the TQ and TD modes are thus expected to slightly deviate from those effectively existing with the experimental permittivity data. Since the real and imaginary parts of the permittivity are both underestimated around $3 \mathrm{eV}$ in our case, the real part of the eigenfrequency would be smaller and the imaginary part larger; that is, the experimental mode is likely to be red-shifted and more lossy.

Pulse Excitation, Linear Regime. We now consider a pulse, centered at $t=0$, with a Gaussian frequency envelope with $\omega_{0}=\operatorname{Re}\left\{\omega_{\mathrm{LD}}\right\}=1.74 \mathrm{eV}$, i.e., centered at the longitudinal dipolar resonance, and width $\Delta \omega=0.071 \mathrm{eV}$, leading to a Gaussian pulse with fwhm $=22$ fs. Figure 3 shows the spectrum and temporal dynamics of the $E_{z}$ component of the electric field, oriented along the nanorod axis, at a distance $R=10 \mu \mathrm{m}$ in the forward direction $(x=0, y=R, z=0)$. Even though the pulse spectral width is smaller than the width of the harmonic response $E_{\mathrm{h}}(\omega)$, the response $E(\omega)=E_{\mathrm{h}}(\omega) S(\omega)$ is indeed narrower than the pulse, Figure 3(a), and must thus correspond to a longer time signature. This is indeed what is observed in the temporal plots shown in Figure 3(b). At all times, the field corresponding to the blue curve shows a $\pi / 2$ phase shift with respect to the driving pulse (black curve); see zoomed-in plot in Figure 3(c,d), a typical behavior for a forced oscillator driven at its resonant frequency. At larger times $t \gtrsim 75 \mathrm{fs,} \mathrm{Figure} \mathrm{3(d),}$ when the amplitude of the excitation pulse becomes negligible, an exponential decay of the oscillating field amplitude is observed, as expected for a free damped harmonic oscillator. In order to confirm that the behavior observed at $t \gtrsim 75 \mathrm{fs}$ is due to the $\mathrm{LD}$ resonance, the field evolution given by eq 2 with the parameters of the LD mode given in Table 1 is superposed to the full wave computations, blue circles in Figure 3(d). The excellent agreement between the full wave computation and the dynamics associated with the LD mode demonstrates that the latter plays the dominant role in the system; furthermore, this demonstrates how the knowledge of the modes supported by a system can be used to reconstruct its dynamics. ${ }^{12}$ The exponential decay envelope is prolonged before $t \approx 78$ fs to clearly show the transition between forced and free regimes. Note that the maximum of the driving pulse indeed occurs at $t$ $=n_{\mathrm{bg}} R / c_{0}=44.36 \mathrm{fs}$, with $c_{0}=299792458 \mathrm{~ms}^{-1}$, Figure 3(b). In addition, it is apparent that the maximum of the field response is delayed relatively to the driving pulse maximum, another well-known feature of harmonic oscillators driven at their resonances; see Figures S1 and S2.

Pulse Excitation, Nonlinear Regime. The nonlinear response of the nanorod is now studied for a pulse with the same width as in the Pulse Excitation, Linear Regime section but centered at $\omega_{0}=\operatorname{Re}\left\{\omega_{\mathrm{LQ}} / 2\right\}=1.4 \mathrm{eV}$, i.e., at half the LQ resonance. This is done to avoid the excitation of higher order modes at the $\mathrm{SH}$ frequencies and thus concentrate only on the LQ and TD mode radiation, which are expected to be the only contributions at the nonlinear frequencies around $2.8 \mathrm{eV}$ in the small-nanoparticle limit; see Figure 2(b). The width of the pulse, $\Delta \omega=0.071 \mathrm{eV}$, further ensures that the contribution of the high-order modes above $\omega_{\mathrm{TD}}$ remains negligible. In Figure 4 we show the $E_{y}$ component of the electric field at a distance $R=$ $10 \mu \mathrm{m}$ and at $\pm 45^{\circ}$ angle in the $O y z$ plane as well as in the $O z$ direction.

The electric fields taken at the $\pm 45^{\circ}$ angles exhibit a clear resonant dynamic with a long lifetime due to the LQ mode; see Table 1 . In the $\mathrm{O} z$ direction $\left(0^{\circ}\right)$, the LQ mode cannot radiate and the field observed (blue curve) is thus solely due to the TD mode. Since the TD mode is driven below its resonant frequency and is relatively lossy, $\tau_{\mathrm{TD}}=3.93 \mathrm{fs}$, only its forced behavior is observed and the temporal response is symmetric with respect to its center. To understand why the TD mode
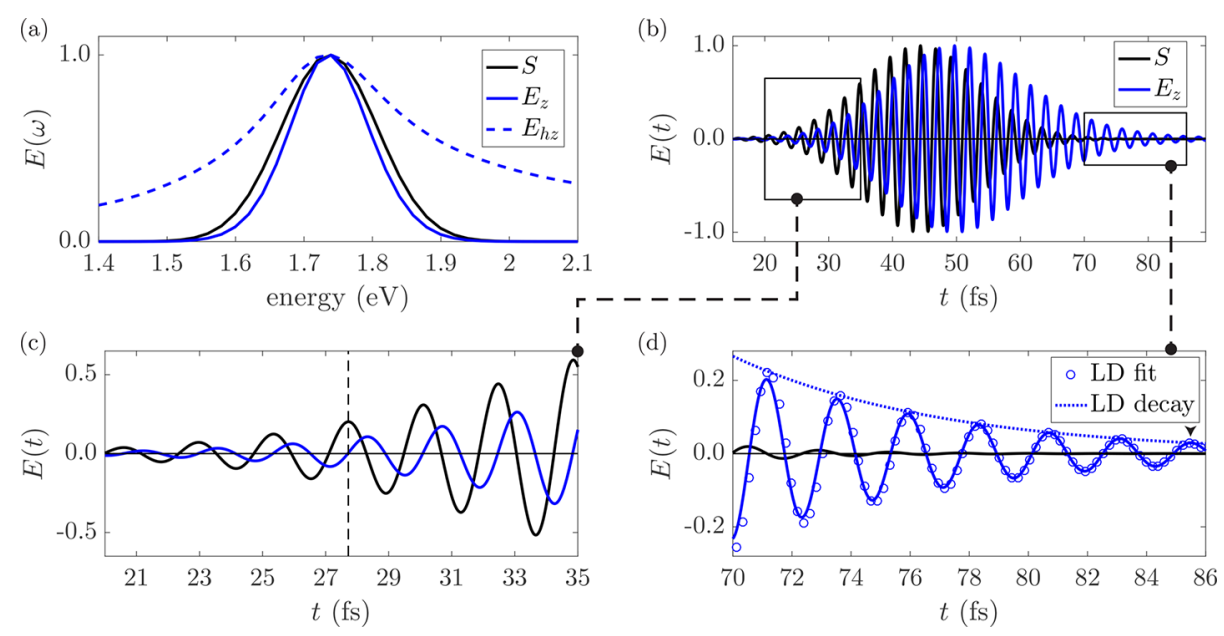

Figure 3. Linear response of the $E_{z}$ component at a distance of $10 \mu \mathrm{m}$ in the forward (positive $y$ ) direction. (a) Spectra obtained with monochromatic plane-wave excitations (dashed blue curve), frequency envelope of the pulse centered at $\omega=1.74 \mathrm{eV}$ with $\Delta \omega=0.071 \mathrm{eV}$ (black curve), and the linear response driven by the pulse (blue curve). (b) Time domain electric field evolutions of the driving pulse (black line) and the linear response (blue curve). (c) Zoom-in on the forced regime. The vertical line highlights the $\pi / 2$ phase shift between the excitation and the response. (d) Zoom-in on the free harmonic motion and fit of the damped harmonic oscillator free response with the parameters of the longitudinal dipolar eigenmode. The black arrowhead indicates where the fit was made. 

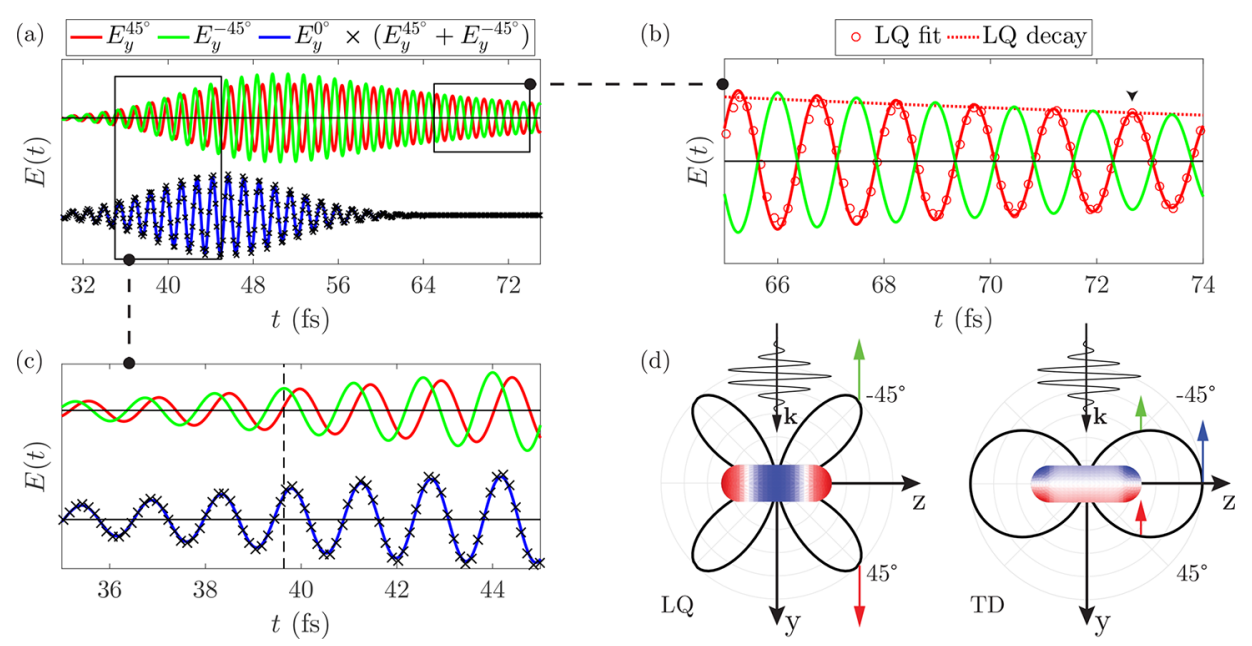

Figure 4. Nonlinear response for a pulse of central frequency $\omega_{0}=1.4 \mathrm{eV}$ and width $\Delta \omega=0.071 \mathrm{eV}$. (a) $E_{y}$ component of the electric field at a distance of $10 \mu \mathrm{m}$. Red, green, and blue curves respectively show the field in the forward $45^{\circ}(z=y)$, backward $-45^{\circ}(z=-y)$, and top $0^{\circ}(z=0)$ directions. The black curve is the sum of the green and red curves. The blue and black curves are vertically shifted for clarity. (b, c) Details of the time evolution shown in (a). The vertical dashed line in (c) highlights the phase difference between the fields. The arrowhead in (b) indicates the time where the fit with the LQ mode was made. (d) Radiation patterns of the LQ and TD modes in the Oyz plane and corresponding qualitative amplitudes of the $E_{y}$ field component at the positions where the fields of panels (a)-(c) are evaluated.

shows a response as strong as the LQ mode even though its resonant frequency is relatively far from $2 \times 1.4=2.8 \mathrm{eV}$, one needs to consider a few points. First, the TD mode is spectrally broad due its large eigenfrequency imaginary part $\left(\operatorname{Im}\left\{\omega_{\mathrm{TD}}\right\}=\right.$ $-0.168 \mathrm{eV}$ ), and since it is a dipolar resonance it is better coupled to the far field than the LQ. Indeed, the multipole decomposition of the SHG spectrum gives a $15 \%$ contribution of the dipole component for a harmonic plane-wave excitation at $2.8 \mathrm{eV}$; see Figure S3. Second, even though the central linear frequency is fixed at $1.4 \mathrm{eV}$, the $\mathrm{LD}$ resonance is effectively blue-shifting the linear response maximum, making the maximum of the nonlinear excitation closer to the TD eigenfrequency. Finally, the linear pulse width is $0.071 \mathrm{eV}$, so that the effective spectral width of the nonlinear excitation $P_{\mathrm{NL}}$ can be expected to be roughly twice this value, making the spectral overlap between $P_{\mathrm{NL}}$ and the TD even greater.

We observe in Figure 4(c) that the electric fields in the forward $\left(45^{\circ}\right.$, red line) and backward $\left(-45^{\circ}\right.$, green line) directions have slightly different amplitudes for $t \lesssim 60$ fs. The difference in the field amplitudes in the $\pm 45^{\circ}$ directions for $t \lesssim$ $60 \mathrm{fs}$ is caused by the interference between the LQ and TD modes, as already reported in the continuous regime in refs 51 and 52. Indeed, the electric field orientation associated with the quadrupolar and dipolar emissions at the two observation points is different, Figure 4(d), and interferes constructively in the backward direction and destructively in the forward direction. Let us note that the constructive/destructive interferences can generally happen in either direction depending on the relative spectral position of the excitation and the LQ and TD modes. ${ }^{52}$ To further study this phenomenon, the sum of the two responses in the $\pm 45^{\circ}$ directions (red+green curves) is computed, and it is observed that the result, black symbols in Figure $4(\mathrm{a}, \mathrm{c})$, fits almost perfectly with the transverse dipolar response taken in the $\mathrm{Oz}$ direction (blue curve). The extremely small discrepancy is fortuitous: it is due to the difference in amplitude of the dipolar and quadrupolar radiations in the $\pm 45^{\circ}$ and $\mathrm{Oz}$ direction as well as to the relative amplitude of each mode. Let us note that a similar interference effect between the TD and LQ modes also exists for the $E_{z}$ component of the fields (data not shown). The temporal overlap of the sum of the two fields in the $\pm 45^{\circ}$ and $\mathrm{Oz}$ directions, respectively due to the LQ and TD modes, thus confirms that the TD resonance is indeed the source of the observed amplitude difference in the $\pm 45^{\circ}$ directions.

For times $t \gtrsim 65$ fs the short-lived TD mode vanishes with the exciting pulse so the interference process disappears and the two fields in the $\pm 45^{\circ}$ directions retrieve the same amplitude, Figure 4(b). As for the linear response in the Pulse Excitation, Linear Regime section, at $t \gtrsim 65 \mathrm{fs}$, the behavior of a free damped harmonic oscillator is observed. The parameters of the LQ mode are used to plot eq 2 (red circles), which fit with great accuracy the computed field evolution in the $+45^{\circ}$ direction, the same agreement being expected in the backward $-45^{\circ}$ direction due to the symmetry of the geometry. We additionally notice that during the time where the interference between the two modes occurs, Figure 4(c), the two fields at $\pm 45^{\circ}$ are not perfectly out of phase as they should be if only the LQ mode was excited, Figure 4(d). This is because the TD and $\mathrm{LD}$ modes do not have the same phase with respect to the nonlinear excitation and thus to each other. The interference created by the TD mode thus modifies the relative phase shift between the fields in the $\pm 45^{\circ}$ directions. When the dipolar mode vanishes, the two fields retrieve their relative $\pi$ phase shift (Figure 4(b)), as expected for a purely quadrupolar radiation. Finally, we observe again a shift between the excitation maximum and the response of the quadrupolar mode. As was the case in the linear regime, we see that the eigenmodes are of prime importance in the study of the spectral and dynamical behavior of the $\mathrm{SH}$ response of plasmonic nanoparticles.

Multipolar Analysis. Finally, we study the nonlinear temporal response of the nanorod for various pulse parameters. Six different pulses at two different central frequencies and with three different widths are considered, viz., $\omega_{0}=1.4$ and $1.74 \mathrm{eV}$; $\Delta \omega=0.071,0.14$, and $0.21 \mathrm{eV}$. The three spectral widths correspond respectively to a temporal fwhm of 22,15 , and $7 \mathrm{fs}$ for the field amplitude, and the effective fwhm for the corresponding intensity is given by fwhm $/ \sqrt{2}$. The central frequencies and widths of the pulses at the fundamental stage 
(a)

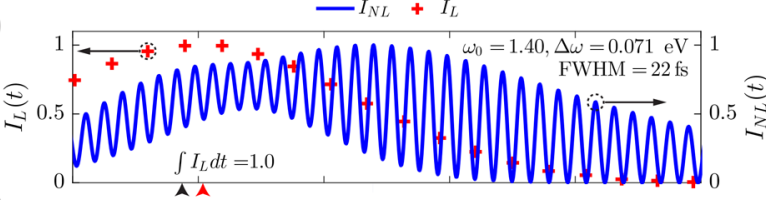

(b)

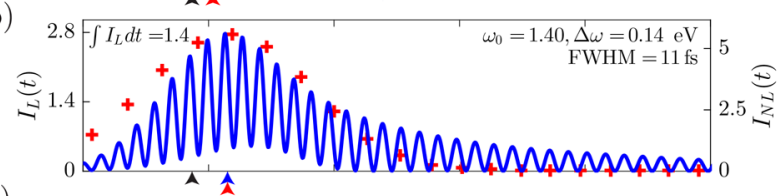

(c)

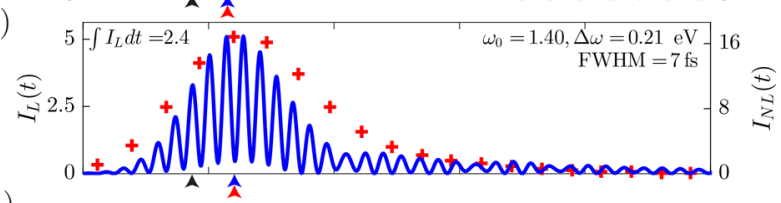

(d)

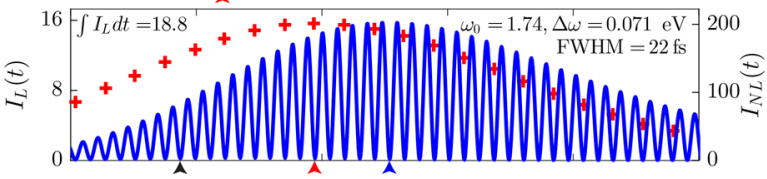

(e)

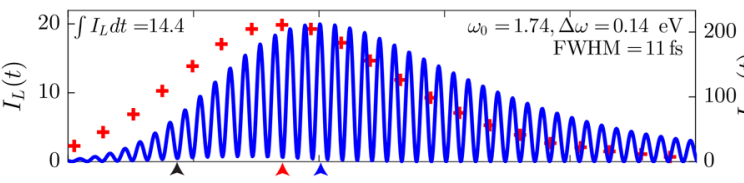

(f)

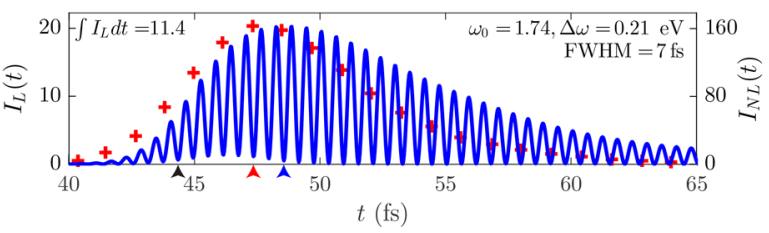

(g)

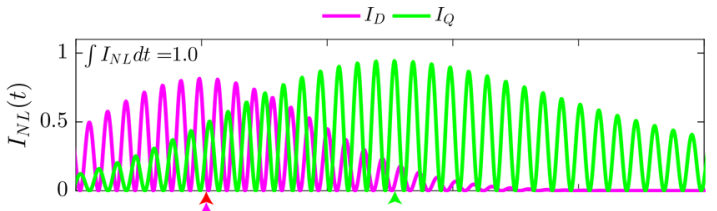

(h)

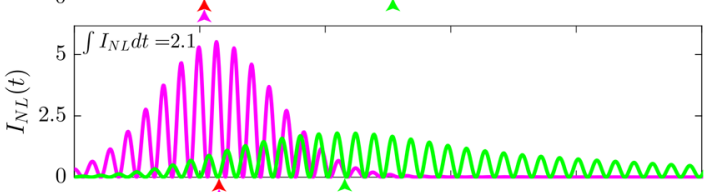

(i)

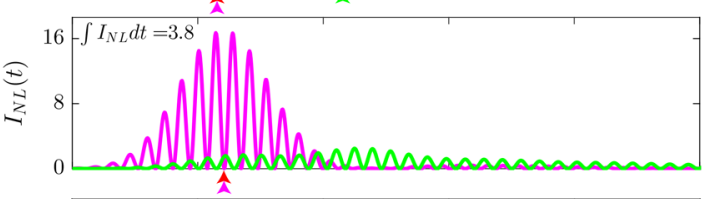

(j)

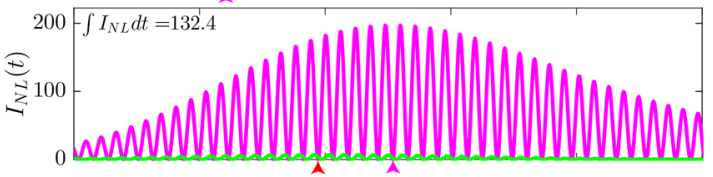

(k)

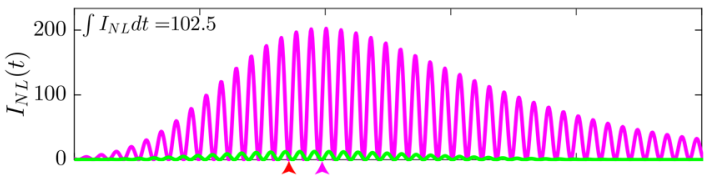

(1)

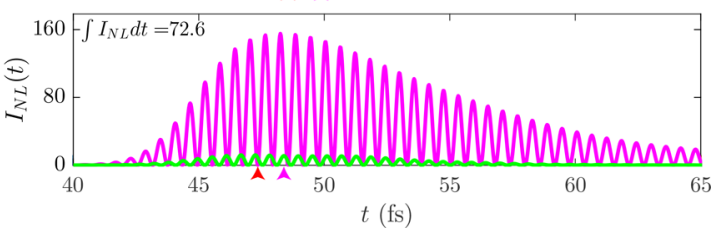

Figure 5. Multipolar analysis of the linear and nonlinear radiations. Six different pulses are considered, at central energies $\omega_{0}=1.4$ and $1.74 \mathrm{eV}$ and widths $\Delta \omega=0.071,0.14$, and $0.28 \mathrm{eV}$. (a-f) Linear (red symbols, value on the left vertical axis) and nonlinear (blue curve, value on the right vertical axis) scattered intensity. The pulse parameters $\left(\omega_{0}, \Delta \omega\right)$ are indicated in the top right corner of each plot. For clarity the linear response is taken only at the maxima of $I_{\mathrm{L}}(t)$. Each linear and respectively nonlinear curve are normalized to the corresponding curve in (a). We observe that both the linear and nonlinear peak intensities are minimal for the pulse parameters used in panel (a). The linear intensity integrated in the range 25 fs $<t<$ $120 \mathrm{fs}$ is indicated in the plots and normalized to the one in (a). The black arrowheads on the horizontal axis indicate the center of the exciting pulse at $44.36 \mathrm{fs}$, whereas the red and blue ones indicate the maximum of the linear and nonlinear responses. ( $\mathrm{g}$, $\mathrm{h}$ ) Corresponding multipolar decompositions of the nonlinear radiations. Multipoles other that electric dipolar and quadrupolar components are found to have negligible contributions. The intensity integrated in the range $25 \mathrm{fs}<t<120 \mathrm{fs}$ is indicated in the top left corner of each plot and normalized to the one in (g). The colored arrowheads on the horizontal axis indicate maxima of the dipolar and quadrupolar components when possible. Note that the indicated fwhm is given for the field, not the intensity.

are shown in Figure 2(b). The corresponding nonlinear central frequencies, obtained by multiplying by 2 each linear one, are also shown as blue dots. Note however that the real excitation of the nonlinear process comes from the linear response and will thus not necessarily have a maximum at $2 \omega_{0}$ nor a Gaussian shape due to the resonant character of the linear response. For each case, we project the time-dependent linear and nonlinear far fields onto the vector spherical harmonics to decompose the radiation into multipole moments. ${ }^{50}$ The results are shown in Figure 5, where the panels (a)-(f) show the linear and nonlinear scattered intensities and the panels $(\mathrm{g})-(\mathrm{l})$ the dipolar and quadrupolar components of the nonlinear scattered intensity. Other multipolar moments are found to be negligible, and the linear response is purely dipolar. To clarify the plot, the linear intensity is shown only at the peak values of the oscillations, indicated by red markers, since the dynamics does not involve any additional effect compared with the ones presented in the Pulse Excitation, Linear Regime section. Additionally, the intensity is integrated over the range $25<t<$ $120 \mathrm{fs}$ for both linear and nonlinear signals and normalized to the weakest case, which is $\omega_{0}=1.40 \mathrm{eV}$ and $\Delta \omega=0.071 \mathrm{eV}$.
The corresponding data are shown in each panel, thus providing the total energy of the radiated fields. Every curve in Figure $5(\mathrm{a}-\mathrm{f})$ is normalized to the maximum intensity of the corresponding curve for the case $\omega_{0}=1.4 \mathrm{eV}$ and $\Delta \omega=0.071$ $\mathrm{eV}$, which gives the weaker linear and nonlinear peak signals. Finally, the exciting pulses are all normalized to have unit energy so that the comparison of the peak and integrated intensities reflects the intrinsic efficiency of the linear and nonlinear signal generations. We recall Parseval's theorem that states that the energy of a signal in the frequency domain is proportional to the energy of the temporal signal, $\int|E(\omega)|^{2} \mathrm{~d} \omega$ $\propto \int|E(t)|^{2} \mathrm{~d} t$, the proportionality constant being dictated by the definition of the Fourier transform. ${ }^{53}$ Thus, the integrated intensities in the time domain can be explained by analyzing the corresponding spectra. Concerning the linear dynamics, every case shows a fully dipolar response (data not shown), as expected from the excitation of the LD mode; see Figure 2. For the pulse centered at $1.4 \mathrm{eV}$, Figure $5(\mathrm{a}-\mathrm{c})$, it appears that the peak and the integrated intensity increase as the pulse shortens. Indeed, the shorter the pulse, the broader the spectrum, and since the central frequency is here below the LD peak, a 
broadening means a better overlap with the LD resonance at $1.74 \mathrm{eV}$ and thus a stronger signal. This better overlap with the resonance also leads to a slight increase in the delay between the driving pulse and the linear response; see the black and red arrowheads, respectively. On the other hand, when the pulse central frequency is tuned to the $\mathrm{LD}$ resonance at $1.74 \mathrm{eV}$, the shorter pulses lead to a weaker integrated signal (compare Figure 5(d-f)), and it additionally appears that the peak intensity slightly increases for shorter pulses. Keeping in mind that each excitation pulse is normalized to have unit energy, the broadening of the spectrum, which is linked to the reduction of the pulse duration, leads to a weaker maximum amplitude of the pulse spectrum that is centered at the LD resonance. This could explain the decrease in integrated intensity, but not the slight increase in peak intensity for shorter pulses. We first recall that the maximum of the temporal signal can be linked to the integrated signal because $E(t=0) \propto \int E(\omega) \mathrm{d} \omega$, which is indeed different from the energy $\int|E(\omega)|^{2} \mathrm{~d} \omega$. To explain the increase in peak intensity that occurs parallel to the decrease in integrated intensity, we multiplied three Gaussians (with the width of the pulses used in this section and having unit energy) with a damped harmonic oscillator spectral line-shape in order to simulate the linear response $E(\omega)$ of the system. We then compared the results of $\int E(\omega) \mathrm{d} \omega$ and $\int|E(\omega)|^{2} \mathrm{~d} \omega$ and found a good qualitative agreement with the previous results, namely, a decrease of the energy and a slight increase of the integrated signal (data not shown). Finally, we observe that for the pulse centered at $1.74 \mathrm{eV}$, Figure $5(\mathrm{~d}-\mathrm{f})$, the linear response delay is the largest, and it decreases for shorter pulses, as expected for a pulse that shortens; see Figure S2. The nonlinear field dynamics is first studied for femtosecond pulses centered at $1.4 \mathrm{eV}$, Figure $5(\mathrm{a}-\mathrm{c}, \mathrm{g}-\mathrm{i})$. In panels $(\mathrm{a})-(\mathrm{c})$, the nonlinear intensity oscillations do not decrease down to zero at short times. This effect is easily understood by looking at the multipolar decomposition, panels $(\mathrm{g})-(\mathrm{i})$. It appears that the dipolar and quadrupolar components, mainly due to the TD and LQ modes, respectively, are out of phase, as explained in the Pulse Excitation, Nonlinear Regime section. When the driving pulse vanishes, only the long-lived quadrupole mode remains and the scattered intensity oscillations can indeed vanish periodically. Indeed, the TD mode has a lifetime of 3.93 fs and thus cannot outlive the exciting pulse. As the pulse shortens and its spectrum broadens, the overlap between the nonlinear excitation spectrum and the TD mode increases. Thus, it appears that the short-lived dipolar component due to the TD mode becomes predominant during the excitation and that the quadrupolar component takes the lead afterward; see panels $(\mathrm{g})-(\mathrm{i})$. The strong intensity difference between the dipolar and quadrupolar components is due to the fact that dipolar modes are intrinsically more efficient to radiate in the far-field and that, as shown in Figure S3, there is a nonnegligible dipolar contribution to the SHG around $2.8 \mathrm{eV}$. Notice the different vertical axis scales that indicate that the quadrupolar component still increases from panels $(\mathrm{g})$ to (i), the maxima of the $I_{Q}$ curve being respectively $1,1.8$, and 2.5. In panel (i), at around $48 \mathrm{fs}$, one can also observe a small dip in the quadrupolar component envelope, caused by the interference between the LQ mode and higher order modes having a quadrupolar far-field response. Additionally, the overall nonlinear response is also stronger in both peak and integrated intensity for shorter pulses, due to the corresponding stronger linear response. When the central pulse energy is resonant with the $\mathrm{LD}$ mode, $\omega_{0}=1.74 \mathrm{eV}$, the nonlinear signal is mainly dipolar, Figure $5(\mathrm{~d}-\mathrm{f}, \mathrm{j}-\mathrm{l})$. Indeed, the nonlinear excitation is now centered around $2 \times 1.74=3.48 \mathrm{eV}$, close to the TD mode at $3.38 \mathrm{eV}$ and far from the LQ mode. Nevertheless, a long-lived oscillation is observed for the two shortest pulses, panels (k) and (l). The decay observed is too slow to be attributed to the TD mode and thus originates from a higher order mode having a nonvanishing dipolar moment in the $\mathrm{Oy}$ direction. As discussed in the Harmonic Response and Eigenmodes section, such high order modes are expected to have resonant frequencies around the nonlinear excitation $P_{\mathrm{NL}}$ in the present case, explaining further the strength of this dipolar component. This is also in agreement with the SHG spectrum shown in Figure S3, where the SHG response is mostly dipolar around the peak at $3.5 \mathrm{eV}$. The total energy of the signals follows the evolution of the linear response; that is, a smaller integrated intensity is obtained for shorter pulses. Overall, the maximum peak and integrated intensities are indeed obtained when the pulse is centered at the longitudinal dipolar resonance mode for both the linear and nonlinear fields. The delayed-response behavior of a mode excited at resonance is also apparent in the nonlinear signals, keeping in mind that the source of the nonlinear signal is the linear response, which is itself delayed with respect to the exciting pulse. For the pulse centered at $1.4 \mathrm{eV}$, the nonlinear excitation $P_{\mathrm{NL}}$ is close to the LQ mode, and thus the delay between the linear response and the quadrupolar component is large and increases with the pulse duration. For the shortest pulse, panel (i), interferences with high-order modes make the measure of the delay not pertinent. On the other hand, the nonlinear excitation is far from the TD mode, leading to a vanishing delay for the dipolar component. Concerning the pulse centered at $1.74 \mathrm{eV}$, the nonlinear excitation is now close to the TD mode, and we observe a delay of the dipolar response that increases with the pulse duration as expected.

\section{CONCLUSION}

The dynamics of the linear and second-order nonlinear fields scattered by a single silver nanorod under femtosecond pulse plane-wave illumination have been studied. The linear response was first presented to establish the method. It was shown that it is indeed possible to observe the dynamics of single eigenmodes that behave like damped harmonic oscillators. Then the nonlinear scattered fields were studied, first in detail for the case where the longitudinal quadrupolar mode is resonant at the nonlinear frequency. It was observed that the transverse dipolar mode interfered with the quadrupolar mode during the time when the excitation pulse is still present, but that only the quadrupolar response remains afterward, due to its longer lifetime. Finally, a multipolar analysis of the scattered field was conducted for two different pulse center energies and three different widths. Different responses are observed depending upon the pulse width and center frequency, like a switch between quadrupolar and dipolar dominant emissions over time as well as changes in the peak intensity value or the total energy radiated. It appears that the modal structure of the nanorod is of high importance to correctly understand the temporal evolution of the nonlinear fields. These results provide new insight into the linear and nonlinear dynamics of localized surface plasmons, as one could tailor the exciting pulse parameters to achieve a variety of dynamical responses. Furthermore, these results are important regarding the accurate measurement of plasmon lifetime with the help of nonlinear optics. ${ }^{16-18}$ Indeed, to do so, one needs to carefully disentangle 
the linear and nonlinear plasmon dynamics as discussed in this article.

\section{METHODS}

To reconstruct a temporal signal from its frequency components, the following inverse Fourier transform is used:

$$
E(t)=\mathcal{F}^{-1}\{E\}(t)=\int_{-\infty}^{\infty} E(\omega) \mathrm{e}^{-i \omega t} \mathrm{~d} \omega
$$

The temporal signal $E(t)$ must be real; hence $E(-\omega)=E^{*}(\omega)$, where $*$ denotes the complex conjugate. Equation 4 then reduces to

$$
E(t)=2 \operatorname{Re}\left\{\int_{0}^{\infty} E(\omega) \mathrm{e}^{-i \omega t} \mathrm{~d} \omega\right\}
$$

A finite amount of frequencies $\omega_{n}$ is computed, and the above integral reduces to a sum. Consequentially $E(t)$ becomes $T$ periodic with $T=2 \pi / \delta \omega, \delta \omega$ being the constant frequency step between each considered frequency $\omega_{n}$. To correctly retrieve the plasmon dynamics, it is mandatory that the periodicity induced by the frequency sampling is sufficiently large compared to the largest plasmon lifetime. Plasmons are known to have lifetimes on the order of femtoseconds, and we choose $\delta \omega=0.02 \mathrm{eV}$, leading to $T=207 \mathrm{fs}$.

To construct the linear and nonlinear temporal signals, the first step is to compute a set of $N$ linear harmonic solutions $E_{\mathrm{h}}\left(\omega_{n}\right)$ at equally spaced frequencies between $\omega_{\mathrm{A}}$ and $\omega_{\mathrm{B}}$, which can then be used to form any signal $E(\omega)$ having a limited bandwidth $\omega_{\mathrm{A}} \leq \omega \leq \omega_{\mathrm{B}}$. $E_{\mathrm{h}}\left(\omega_{n}\right)$ effectively acts as a transfer function, so that the response $E\left(\omega_{n}\right)$ of the nanorod to an excitation having the spectrum $S\left(\omega_{n}\right)$ is $E\left(\omega_{n}\right)=E_{\mathrm{h}}\left(\omega_{n}\right)$ $S\left(\omega_{n}\right)$, meaning that each frequency component $E_{\mathrm{h}}\left(\omega_{n}\right)$ is weighted according to the function $S\left(\omega_{n}\right)$. One then has to make a second-order computation for each possible pair of frequencies $\left(\omega_{m}, \omega_{n}\right)$ composing the femtosecond pulse, taking into account the different weights of each component:

$$
\begin{aligned}
E\left(\omega_{m}+\omega_{n}\right) & =\quad E\left(\omega_{m}\right) E\left(\omega_{n}\right) \\
& =E_{\mathrm{h}}\left(\omega_{m}\right) S\left(\omega_{m}\right) E_{\mathrm{h}}\left(\omega_{n}\right) S\left(\omega_{n}\right)
\end{aligned}
$$

and adding accordingly the pairs that give the same frequency. The total number of combinations is $N^{2}$ with $N$ SHG and $\left(N^{2}\right.$ $-N)$ SFG combinations. Since the process $\left(\omega_{m}, \omega_{n}\right)$ gives the same nonlinear response as $\left(\omega_{n}, \omega_{m}\right)$, only half of the SFG computations have to be performed. Thus, the total number of required nonlinear computations is $N+\left(N^{2}-N\right) / 2=\left(N^{2}+\right.$ $N) / 2$. The temporal responses, both linear and nonlinear, are obtained using eq 5 .

\section{ASSOCIATED CONTENT}

\section{S Supporting Information}

The Supporting Information is available free of charge on the ACS Publications website at DOI: 10.1021/acsphotonics.8b00479.

Figures detailing the dynamical response of a damped harmonic oscillator to Gaussian pulses and multipole decomposition of the SHG spectrum (PDF)

\section{AUTHOR INFORMATION}

\section{Corresponding Author}

*E-mail: gabriel.bernasconi@epfl.ch.

\section{ORCID}

Gabriel D. Bernasconi: 0000-0001-7183-9178

Jérémy Butet: 0000-0001-9598-9074

Olivier J. F. Martin: 0000-0002-9574-3119

Notes

The authors declare no competing financial interest.

\section{ACKNOWLEDGMENTS}

Funding from the Swiss National Science Foundation (project 200020_153662) and from the European Research Council (ERC-2015-AdG-695206 Nanofactory) is gratefully acknowledged.

\section{REFERENCES}

(1) Barnes, W. L.; Dereux, A.; Ebbesen, T. W. Surface plasmon subwavelength optics. Nature 2003, 424, 824.

(2) Schuller, J. A.; Barnard, E. S.; Cai, W.; Jun, Y. C.; White, J. S.; Brongersma, M. L. Plasmonics for extreme light concentration and manipulation. Nat. Mater. 2010, 9, 193.

(3) Kottmann, J. P.; Martin, O. J.; Smith, D. R.; Schultz, S. Dramatic localized electromagnetic enhancement in plasmon resonant nanowires. Chem. Phys. Lett. 2001, 341, 1-6.

(4) Liz-Marzán, L. M. Tailoring Surface Plasmons through the Morphology and Assembly of Metal Nanoparticles. Langmuir 2006, $22,32-41$.

(5) Halas, N. J.; Lal, S.; Chang, W.-S.; Link, S.; Nordlander, P. Plasmons in Strongly Coupled Metallic Nanostructures. Chem. Rev. 2011, 111, 3913-3961.

(6) Sönnichsen, C.; Franzl, T.; Wilk, T.; von Plessen, G.; Feldmann, J.; Wilson, O.; Mulvaney, P. Drastic Reduction of Plasmon Damping in Gold Nanorods. Phys. Rev. Lett. 2002, 88, 077402.

(7) Stockman, M. I.; Faleev, S. V.; Bergman, D. J. Coherent Control of Femtosecond Energy Localization in Nanosystems. Phys. Rev. Lett. 2002, 88, 067402.

(8) Aeschlimann, M.; Bauer, M.; Bayer, D.; Brixner, T.; García de Abajo, F. J.; Pfeiffer, W.; Rohmer, M.; Spindler, C.; Steeb, F. Adaptive subwavelength control of nano-optical fields. Nature 2007, 446, 301.

(9) Lévêque, G.; Martin, O. J. F. Narrow-Band Multiresonant Plasmon Nanostructure for the Coherent Control of Light: An Optical Analog of the Xylophone. Phys. Rev. Lett. 2008, 100, 117402.

(10) Accanto, N.; Nieder, J. B.; Piatkowski, L.; Castro-Lopez, M.; Pastorelli, F.; Brinks, D.; van Hulst, N. F. Phase control of femtosecond pulses on the nanoscale using second harmonic nanoparticles. Light: Sci. Appl. 2014, 3, e143.

(11) Piatkowski, L.; Accanto, N.; van Hulst, N. F. Ultrafast Meets Ultrasmall: Controlling Nanoantennas and Molecules. ACS Photonics 2016, 3, 1401-1414.

(12) Faggiani, R.; Losquin, A.; Yang, J.; Mårsell, E.; Mikkelsen, A.; Lalanne, P. Modal Analysis of the Ultrafast Dynamics of Optical Nanoresonators. ACS Photonics 2017, 4, 897-904.

(13) Mårsell, E.; Losquin, A.; Svärd, R.; Miranda, M.; Guo, C.; Harth, A.; Lorek, E.; Mauritsson, J.; Arnold, C. L.; Xu, H.; L'Huillier, A.; Mikkelsen, A. Nanoscale Imaging of Local Few-Femtosecond NearField Dynamics within a Single Plasmonic Nanoantenna. Nano Lett. 2015, 15, 6601-6608.

(14) Razinskas, G.; Kilbane, D.; Melchior, P.; Geisler, P.; Krauss, E.; Mathias, S.; Hecht, B.; Aeschlimann, M. Normal-Incidence PEEM Imaging of Propagating Modes in a Plasmonic Nanocircuit. Nano Lett. 2016, 16, 6832-6837.

(15) Sun, Q.; Yu, H.; Ueno, K.; Kubo, A.; Matsuo, Y.; Misawa, H. Dissecting the Few-Femtosecond Dephasing Time of Dipole and Quadrupole Modes in Gold Nanoparticles Using Polarized Photoemission Electron Microscopy. ACS Nano 2016, 10, 3835-3842.

(16) Lamprecht, B.; Leitner, A.; Aussenegg, F. Femtosecond decaytime measurement of electron-plasma oscillation in nanolithographically designed silver particles. Appl. Phys. B: Lasers Opt. 1997, 64, 269-272. 
(17) Lamprecht, B.; Leitner, A.; Aussenegg, F. SHG studies of plasmon dephasing in nanoparticles. Appl. Phys. B: Lasers Opt. 1999, 68, 419-423.

(18) Berweger, S.; Atkin, J. M.; Xu, X. G.; Olmon, R. L.; Raschke, M. B. Femtosecond Nanofocusing with Full Optical Waveform Control. Nano Lett. 2011, 11, 4309-4313.

(19) Davidson, R. B.; Yanchenko, A.; Ziegler, J. I.; Avanesyan, S. M.; Lawrie, B. J.; Haglund, R. F. Ultrafast Plasmonic Control of Second Harmonic Generation. ACS Photonics 2016, 3, 1477-1481.

(20) Zentgraf, T.; Christ, A.; Kuhl, J.; Giessen, H. Tailoring the Ultrafast Dephasing of Quasiparticles in Metallic Photonic Crystals. Phys. Rev. Lett. 2004, 93, 243901.

(21) Hanke, T.; Cesar, J.; Knittel, V.; Trügler, A.; Hohenester, U.; Leitenstorfer, A.; Bratschitsch, R. Tailoring Spatiotemporal Light Confinement in Single Plasmonic Nanoantennas. Nano Lett. 2012, 12, 992-996.

(22) Kauranen, M.; Zayats, A. V. Nonlinear plasmonics. Nat. Photonics 2012, 6, 737.

(23) Butet, J.; Brevet, P.-F.; Martin, O. J. F. Optical Second Harmonic Generation in Plasmonic Nanostructures: From Fundamental Principles to Advanced Applications. ACS Nano 2015, 9, 10545-10562.

(24) Canfield, B. K.; Husu, H.; Laukkanen, J.; Bai, B.; Kuittinen, M.; Turunen, J.; Kauranen, M. Local Field Asymmetry Drives SecondHarmonic Generation in Noncentrosymmetric Nanodimers. Nano Lett. 2007, 7, 1251-1255.

(25) Butet, J.; Duboisset, J.; Bachelier, G.; Russier-Antoine, I.; Benichou, E.; Jonin, C.; Brevet, P.-F. Optical Second Harmonic Generation of Single Metallic Nanoparticles Embedded in a Homogeneous Medium. Nano Lett. 2010, 10, 1717-1721.

(26) Zhang, Y.; Grady, N. K.; Ayala-Orozco, C.; Halas, N. J. ThreeDimensional Nanostructures as Highly Efficient Generators of Second Harmonic Light. Nano Lett. 2011, 11, 5519-5523.

(27) Slablab, A.; Xuan, L. L.; Zielinski, M.; de Wilde, Y.; Jacques, V.; Chauvat, D.; Roch, J.-F. Second-harmonic generation from coupled plasmon modes in a single dimer of gold nanospheres. Opt. Express 2012, 20, 220-227.

(28) Kim, M.-K.; Sim, H.; Yoon, S. J.; Gong, S.-H.; Ahn, C. W.; Cho, Y.-H.; Lee, Y.-H. Squeezing Photons into a Point-Like Space. Nano Lett. 2015, 15, 4102-4107.

(29) Metzger, B.; Gui, L.; Fuchs, J.; Floess, D.; Hentschel, M.; Giessen, H. Strong Enhancement of Second Harmonic Emission by Plasmonic Resonances at the Second Harmonic Wavelength. Nano Lett. 2015, 15, 3917-3922.

(30) Gennaro, S. D.; Rahmani, M.; Giannini, V.; Aouani, H.; Sidiropoulos, T. P. H.; Navarro-Cía, M.; Maier, S. A.; Oulton, R. F. The Interplay of Symmetry and Scattering Phase in Second Harmonic Generation from Gold Nanoantennas. Nano Lett. 2016, 16, 52785285.

(31) Thyagarajan, K.; Rivier, S.; Lovera, A.; Martin, O. J. Enhanced second-harmonic generation from double resonant plasmonic antennae. Opt. Express 2012, 20, 12860-12865.

(32) Aouani, H.; Navarro-Cia, M.; Rahmani, M.; Sidiropoulos, T. P. H.; Hong, M.; Oulton, R. F.; Maier, S. A. Multiresonant Broadband Optical Antennas As Efficient Tunable Nanosources of Second Harmonic Light. Nano Lett. 2012, 12, 4997-5002.

(33) Celebrano, M.; Wu, X.; Baselli, M.; Großmann, S.; Biagioni, P.; Locatelli, A.; De Angelis, C.; Cerullo, G.; Osellame, R.; Hecht, B.; Duò, L.; Ciccacci, F.; Finazzi, M. Mode matching in multiresonant plasmonic nanoantennas for enhanced second harmonic generation. Nat. Nanotechnol. 2015, 10, 412.

(34) Ethis de Corny, M.; Chauvet, N.; Laurent, G.; Jeannin, M.; Olgeirsson, L.; Drezet, A.; Huant, S.; Dantelle, G.; Nogues, G.; Bachelier, G. Wave-Mixing Origin and Optimization in Single and Compact Aluminum Nanoantennas. ACS Photonics 2016, 3, 18401846.

(35) Yang, K.-Y.; Butet, J.; Yan, C.; Bernasconi, G. D.; Martin, O. J. F. Enhancement Mechanisms of the Second Harmonic Generation from
Double Resonant Aluminum Nanostructures. ACS Photonics 2017, 4, $1522-1530$.

(36) Butet, J.; Dutta-Gupta, S.; Martin, O. J. F. Surface secondharmonic generation from coupled spherical plasmonic nanoparticles: Eigenmode analysis and symmetry properties. Phys. Rev. B: Condens. Matter Mater. Phys. 2014, 89, 245449.

(37) Bernasconi, G. D.; Butet, J.; Martin, O. J. F. Mode analysis of second-harmonic generation in plasmonic nanostructures. J. Opt. Soc. Am. B 2016, 33, 768.

(38) Smirnova, D.; Smirnov, A. I.; Kivshar, Y. S. Multipolar SecondHarmonic Generation by Mie-Resonant Dielectric Nanoparticles. Phys. Rev. A: At., Mol., Opt. Phys. 2018, 97 DOI: 10.1103/PhysRevA.97.013807.

(39) Kern, A. M.; Martin, O. J. F. Surface integral formulation for 3D simulations of plasmonic and high permittivity nanostructures. J. Opt. Soc. Am. A 2009, 26, 732-740.

(40) Boyd, R. W. Nonlinear Optics; Academic Press, 2003.

(41) Krause, D.; Teplin, C. W.; Rogers, C. T. Optical Surface Second Harmonic Measurements of Isotropic Thin-Film Metals: Gold, Silver, Copper, Aluminum, and Tantalum. J. Appl. Phys. 2004, 96, 36263634.

(42) Wang, F. X.; Rodríguez, F. J.; Albers, W. M.; Ahorinta, R.; Sipe, J. E.; Kauranen, M. Surface and bulk contributions to the second-order nonlinear optical response of a gold film. Phys. Rev. B: Condens. Matter Mater. Phys. 2009, 80, 233402.

(43) Bachelier, G.; Butet, J.; Russier-Antoine, I.; Jonin, C.; Benichou, E.; Brevet, P.-F. Origin of optical second-harmonic generation in spherical gold nanoparticles: Local surface and nonlocal bulk contributions. Phys. Rev. B: Condens. Matter Mater. Phys. 2010, 82, 235403.

(44) Mäkitalo, J.; Suuriniemi, S.; Kauranen, M. Boundary element method for surface nonlinear optics of nanoparticles. Opt. Express 2011, 19, 23386-23399.

(45) Butet, J.; Gallinet, B.; Thyagarajan, K.; Martin, O. J. F. Secondharmonic generation from periodic arrays of arbitrary shape plasmonic nanostructures: a surface integral approach. J. Opt. Soc. Am. B 2013, 30, 2970-2979.

(46) Dadap, J. I.; Shan, J.; Eisenthal, K. B.; Heinz, T. F. SecondHarmonic Rayleigh Scattering from a Sphere of Centrosymmetric Material. Phys. Rev. Lett. 1999, 83, 4045-4048.

(47) Dadap, J. I.; Shan, J.; Heinz, T. F. Theory of optical secondharmonic generation from a sphere of centrosymmetric material: small-particle limit. J. Opt. Soc. Am. B 2004, 21, 1328-1347.

(48) Bai, Q.; Perrin, M.; Sauvan, C.; Hugonin, J.-P.; Lalanne, P. Efficient and intuitive method for the analysis of light scattering by a resonant nanostructure. Opt. Express 2013, 21, 27371.

(49) Johnson, P. B.; Christy, R. W. Optical Constants of the Noble Metals. Phys. Rev. B 1972, 6, 4370-4379.

(50) Mühlig, S.; Menzel, C.; Rockstuhl, C.; Lederer, F. Multipole analysis of meta-atoms. Metamaterials 2011, 5, 64-73.

(51) Butet, J.; Raziman, T.; Yang, K.-Y.; Bernasconi, G. D.; Martin, O. J. Controlling the nonlinear optical properties of plasmonic nanoparticles with the phase of their linear response. Opt. Express 2016, 24, 17138.

(52) Butet, J.; Bernasconi, G. D.; Petit, M.; Bouhelier, A.; Yan, C.; Martin, O. J. F.; Cluzel, B.; Demichel, O. Revealing a Mode Interplay That Controls Second-Harmonic Radiation in Gold Nanoantennas. ACS Photonics 2017, 4, 2923-2929.

(53) Arfken, G. B.; Weber, H.-J.; Harris, F. E. Mathematical Methods for Physicists: A Comprehensive Guide; Academic Press, 2013. 